\begin{tabular}{|c|c|}
\hline 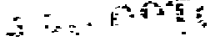 & SLAC/AP--73 \\
\hline & DER9 007733 \\
\hline
\end{tabular}

\title{
COMPUTER AIDED DESIGN OF THREE-DIMENSIONAL WAVEGUIDE LOADED CAVTTIES*
}

\author{
Yehuda Gohen and David U. L. Yu \\ DULY Consultants \\ 1912 MacArthur Street \\ Rancho Palos Verdes, California 90732 \\ and \\ Stanford Linear Accelerator Center \\ Stanford University, Stanford, California 94309
}

Three-dimensional electromagnetic codes which solve Maxwell's equations in the frequency domain are powerful numerical tools in design of RF cavities. Frequencies of TE and TM modes as well as values of $R / Q$ and $Q$ due to wall losses can be calculated accurately with codes such as MAFIA. ${ }^{\dagger}$ The major limitation of these codes is their inability to calculate direct!y the electromagnetic behavior of externally loaded cavities since there can be no net energy flow across the boundaries of the model structure. Time domain 3-D codes are capable of calculating output power flow into external waveguide, but at the expense of extremely long run time.

We have developed two simple methods to calculate the power transport through an external waveguide of a loadsd cavity utilizing the RF parameters obtained from the frequency domain codes. In the first method the external power loss through an open waveguide is expressed in terms of equivalent circuit coupling parameters between a closed waveguide and a cavity to which it is connected. As we shall see, this equivalent circuit approach is limited in its applicability only to structures with high loaded $Q$ values, say, $Q_{L}<200$. In the second method, the power flow through an external waveguide is calculated from an analysis of the electromagnetic field components of the standing waves in the closed waveguide-cavity structure. Our models make use of the MAFIA code to obtain values of structural parameters and fields when an external waveguide is abruptly terminated with a metal surface. A typical model consists of an output cavity attached via an iris to a short waveguide section ended with a conducting cap. Our methodologies of calculating the loaded $Q_{L}$ in terms of the parameters of the closed waveguide-cavity model are described in the following sections. We have obtained reasonable and encouraging results for several loaded cavities whose values of $Q_{L}$ have been experimentally measured. Of

* Work supported by the Department of Energy under contract DE-AC03-87ER80529 and in part by DE-AC03-36SF00515.

† R. Katt et al., Proceedinge of the 1986 Linear Accelerator Conference, SLAC-Report-303 (September 1986), pp. 276-278. 
particulr interest is the application to low- $Q$ structures such as the rejativistic klystron output ravities. The electromagnetic field method yields good agreement with experimental measurements. This method has also been successiully applied ${ }^{\ddagger}$ to high gradient accelerating structures with slots for damping out higher modes as recently proposed by Palmer.

\section{Equivalent Circuit Approach}

The main assumption in this model is that a closed cavity-waveguide structure can be considered as two separate equivalent circuits, each with a capacitor $C_{i}$, an inductor $L_{i}$, and a series resistor $R_{i}$, which describes the wall losses. When the iris connecting the cavity and the waveguide is opened, the two circuits are combined and coupled through a mutual inductance $(m)$ and a mutual capacitance $(c)$ which together account for the gap voltage across the iris. This equivalent circuit is shown in Fig. 1. The circuit parameters for a given mode can be related to the frequency, shunt impedance $R$ and $R / Q$ of the closed cavity-waveguide structure. The cavity parameters are defined in the usual way by:

$$
\begin{aligned}
Q & =\frac{\omega U}{P} \\
R & =\frac{\left|\int E_{z}(x, y, z) \exp (i \omega z / v) d z\right|^{2}}{2 P}, \\
\frac{R}{Q} & =\frac{\left|\int E_{z}(x, y, z) \exp (i \omega z / v) d z\right|^{2}}{2 \omega U},
\end{aligned}
$$

where $\omega$ is the frequency of a given mode, $U$ is the stored energy, $P$ is the power dissipation due to wall losses, $v$ is the speed of the particles passing through the cavity, $E_{z}$ is the component of the electric field along the direction of the beam.

In order to calculate $m$, we run MAFIA with a finite closed section of the waveguide attached to the cavity via an iris. Neglecting the series resistances $R_{i}$ which are much smaller than all the other impedances, we find the resonant frequency $\omega_{R}$ for the equivalent circuit shown in Fig. 1 by the relation:

$$
\left(\omega_{R} L_{1}-\frac{1}{\omega_{R} C_{1}}\right)\left(\omega_{R} L_{2}-\frac{1}{\omega_{R} C_{2}}\right)=\left(\omega_{R} m\right)^{2}
$$

or

$$
\omega_{R}=\omega_{1}\left\{1+\left(\frac{1}{2}\right)\left(\frac{m^{2}}{L_{1} L_{2}}\right)\left[\frac{\omega_{1}^{2}}{\left(\omega_{1}^{2}-\omega_{2}^{2}\right)}\right]\right\}
$$

ID. Yu and Y. Goren, to be published.

f R. B. Palmer, SLAC-PUB-4542, July 1988

D. Yu/January 1989 
for $m^{2} \ll L_{1} L_{2}$. In Eq. $5, w_{1}$ and $w_{2}$ are the frequencies of the respective circuits of the cavity and waveguide. Also, we have assumed that mutual inductance is the dominant coupling. The circuit parameters $L_{1}$ and $C_{1}$ of the cavity are related to the frequency and $(R / Q)_{1}$ by:

$$
\omega_{1}=\left(L_{1} C_{1}\right)^{-1 / 2}
$$

and

$$
\left(\frac{R}{Q}\right)_{1}=\left(\frac{L_{1}}{C_{1}}\right)^{1 / 2}
$$

For a waveguide with dimensions $a, b$ and $h$ shown in Fig. 2, the resonant frequency for a full wavelength $\mathrm{TE}_{102}$ mode is given by:

$$
\omega_{2}=\pi c\left(\frac{4}{h^{2}}+\frac{1}{b^{2}}\right)^{1 / 2}
$$

where $c$ is the speed of light in vacuum. $R / Q$ for the waveguide is given by:

$$
\left(\frac{R}{Q}\right)_{2}=\left(\frac{4}{\pi}\right)\left(\frac{\mu_{0}}{\epsilon_{0}}\right)^{1 / 2}\left[\frac{a}{\left(4 b^{2}+h^{2}\right)^{1 / 2}}\right],
$$

where $\mu_{0}$ and $\epsilon_{0}$ are respectively the permeability and permittivity in free space. Obtaining $\omega_{R}$ from the MAFIA calculation for the closed cavity-waveguide structure, we can solve for the mutual inductance $m$ using Eq. 5 .

When the end of the waveguide is opened to allow the outflow of power, the equivalent circuit of the loaded cavity is shown in Fig. 3 , where $Z_{L}$ is the impedance of a matched load at the end of the open waveguide. If we assume the circuit parameters depend only very weakly on frequency, i.e., the mutual inductance $m$ remains approximately the same when the waveguide is open, the loaded $Q_{L}$ value can be calculated by:

$$
Q_{L}=\left(\frac{L_{l}}{C_{1}}\right)^{1 / 2}\left[R_{1}+\frac{(\omega m)^{2}}{\bar{Z}_{L}}\right]^{-1}
$$

As an example, we can estimate $Q_{L}$ for the cavity-waveguide structure shown in Fig. 4 with the simple method just outlined. The closed cavity parameters calculated from MAFIA are $f_{R}=11.341 \mathrm{GHz}, Q=3.672 \times 10^{3}, R$ (shunt) $=5.37 \times 10^{5} \Omega$, and $R / Q=146.21 \Omega$.

Using these numbers the cavity inductance is calculated from Eqs. 5 and 6 to be $L_{1}=(R / Q) / \omega_{R}=1.97 \times 10^{-9} \mathrm{H}$. 
For a finite section of a rectangular WR90 waveguide with the dimensions $a=$ $1.016 \mathrm{~cm}, b=2.286 \mathrm{~cm}$, and $h=2.488 \mathrm{~cm}$, we find $f_{2}=13.727 \mathrm{GHz}$, and $L_{2}=$ $1.086 \times 10^{-9} \mathrm{H}$.

Substituting the above numbers into Eq. 5, we calculate the mutual inductance to be $m=2.42 \times 10^{-10} \mathrm{H}$.

The matched impedance of the WR90 waveguide is given by:

$$
Z_{\mathrm{TE}_{102}}=\frac{\left(\mu_{0} / \epsilon_{0}\right)^{1 / 2}\left(2 f_{2} / c\right)}{\left[\left(2 f_{2} / c\right)^{2}-\left(1 / b^{2}\right)\right]^{1 / 2}},
$$

Using Eq. 10 we find the loaded $Q_{L}$ value of the structure to be 210 . In the next section we will use a completely different and perhaps more physically intuitive method of calculating the loaded $Q$ value. For comparison, the loaded $Q_{L}$ from the electromagnetic field approach is 220 , which differs only by $5 \%$ from the equivalent circuit approach.

A criterion for the accuracy of the equivalent circuit approach can be obtained by calculating $R / Q$ from the expression:

$$
\left(\frac{R}{Q}\right)^{-1}=\omega_{R} C_{1}\left\{1+\left[\frac{\left(\omega_{2} / \omega_{1}\right)^{2}\left(\omega_{R}^{2}-\omega_{1}^{2}\right)}{\left(\omega_{R}^{2}-\omega_{2}^{2}\right)}\right]\right\}
$$

Equation (12) does not contain explicitly the coupling parameter $m$. By comparing the MAFIA result of $R / Q$ for the cavity-waveguide structure with Eq. (12), an estimate of the degree of consistency can be made. For the example of Fig. 4 considered above, the values of $R / Q$ from these two calculations are:

$$
\begin{aligned}
\text { MAFIA }: & R / Q=128.0 \\
\text { Eq. }(9): & R / Q=118.0
\end{aligned}
$$

or about $8 \%$ difference. This indicates that the equivalent circuit approach is a good approximation for the higly- $Q$ cavity shown in Fig. 4.

\section{Electromagnetic Field Approach}

The $Q$ value of a waveguide loaded cavity can be found once the eluctric field of the outgoing travelling wave is determined. As noted earlier, however, 3.D frequency domain codes are not capable of calculating the outgoing fields at the waveguide directly. Rather than showing an outgoing travelling wave, MAFIA code typically calculates a standing wave pattern in the waveguide because of the reflective metallic boundary at its end. We propose herein a method which relates the outgoing electric field for an open waveguide witl the stunding wave pattern for a closed waveguide. 
To estimate these fields, we attach to the cavity a section a waveguide longer than a half wavelength, i.e.,

$$
h>\frac{\lambda}{2} \text {. }
$$

A typical standing wave pattern of the axial field $E_{z}$ along the axis of the waveguide is shown in Fig. 5. We note in this figure the finite value of $E_{z}$ at the iris, or entrance from the cavity to the waveguide, and the zero of $E_{z}$ at the closed end, $y=h$. At $y=y_{1}$, the field has a local maximum $E_{z}=E_{\max }$, half of which is going forward with an amplitude $E_{x f}$, and half backward with $E_{x b}$ :

$$
E_{z f}=E_{z b}=\frac{1}{2} E_{\max }
$$

These forward and backward fields are the result of multiple reflertions between the end of the waveguide and the cavity. Let us assume that the dominant reflection at the cavity side is at the iris opening. We can express the forward wave $E_{z f}$ at $y=y_{1}$ by the outgoing electric field $I$ :

$$
E_{z f} \exp \left(i k y_{1}\right)=\frac{I}{1+\rho \exp (-2 i \phi)}
$$

where $\rho$ is the reflection coefficient at the iris, $k=2 \pi / \lambda$ is the wave number and $\phi=k h$ is the phase shift in a single transition of the wave along the waveguide section. Equation (13) expresses the forward electric field $E_{z} f$ at $y=y_{1}$ as a function of two unknowns: the outgoing electric field $I$ and the iris reflection coefficient $\rho$. To find these varjables, we shall assume that they are not strongly frequency dependent around the cavity resonance frequency. Since each MAFIA run gives only one value of $E_{\text {max }}$, we nced two runs with slightly difierent waveguide heights in order to solve for the two variables $I$ and $\rho$. Utilizing Eq. (13) for the two cases, we can write the outgoing electric field $I$ as:

$$
I=\frac{E_{z f 1} \exp \left(i k_{1} y_{1}\right)\left\{1-\exp \left[2 i\left(\phi_{2}-\phi_{1}\right)\right]\right\}}{1-\left(E_{z f 1} / E_{z f 2}\right) \exp \left[2 i\left(\phi_{2}-\phi_{1}\right)\right] \exp \left[i\left(k_{1} y_{1}-k_{2} y_{2}\right)\right]}
$$

where the indices 1 and 2 refer to two parameters obtained from two different MAFIA runs with waveguide heights of $h_{1}$ and $h_{2}$ respectively. Making use of the relation:

$$
\frac{\lambda_{j}}{4}=h_{j}-y_{j} \quad j=1,2
$$

and normalizing the fields with the potential:

$$
V=\int_{-\infty}^{\infty} E_{z}(z, r=0) d z
$$


we obtain the final expression for the normalized outgoing electric field:

$$
\hat{I}=\frac{-i \widehat{E}_{z f 1} \exp \left(i \phi_{1}\right)\left\{1-\exp \left[2 i\left(\phi_{2}-\phi_{1}\right)\right]\right\}}{1-\left(\hat{E}_{z f 1} / \hat{E}_{z f 2}\right) \exp \left[i\left(\phi_{2}-\phi_{1}\right)\right]}
$$

where the ${ }^{\wedge}$ symbol on variable $X$ indicates the normalization by the voltage:

$$
\hat{X}=\frac{X}{V}
$$

The shunt resistance $\boldsymbol{R}$ related to the outgoing power flow can be calculated from:

$$
R^{-1}=\frac{1}{2} \frac{\widehat{I}^{2}}{\mu_{0}} \frac{k}{\omega} a b
$$

where we assume that the power flow is in the $\mathrm{TE}_{01}$ mode. The loaded shunt resistance $R_{L}$ is given by

$$
\frac{1}{R_{L}}=\frac{1}{R}+\frac{1}{R_{c}}
$$

where $R_{r}$ is the wall loss shunt resistance of the closed cavity.

The waveguide loaded $Q$-value, $Q_{L}$, can be obtained from:

$$
Q_{L}=\frac{R_{L}}{(R / Q)_{e}}
$$

where $(R / Q)_{c}$ is the value of $R / Q$ for the closed cavity.

To demonstrate the above algorithm, we first return to the high- $Q$ cavity shown in Fig. 4. Terminating the waveguide $2.49 \mathrm{~cm}$ away from the iris opening, we use MAFIA to calculate the electric fields along the waveguide axis, and the results are shown in Fig. 6. From this run we calculate $E_{x f 1}$ and $\phi_{1}$ to be:

$$
E_{z f 1}=-5.5523 \mathrm{~m}^{-1}
$$

and

$$
\phi_{t}=4.8246 \mathrm{rad} .
$$

Adding $0.25 \mathrm{~cm}$ extension to the waveguide and running the program again we obtain:

$$
\begin{aligned}
E_{x f 2} & =-6.9050 \mathrm{~m}^{-1} \\
\phi_{2} & =5.2836 \mathrm{rad} .
\end{aligned}
$$

From Eq. (17), the outgoing electric field is calculated to be $I=-10.7877 \mathrm{~m}^{-1}$. Substituting this result together with the frequency $\omega$ and wave number $k$ into Eq. (19), 
we obtain the power flow shunt resistance to be $R=3.4185 \times 10^{4} \Omega$. The closed cavity shunt resistance is given by MAFIA to be $R_{c}=5.3690 \times 10^{5} \Omega$. The loaded shunt resistance is calculated with $\mathrm{Eq}_{q}$. (20) to be $R_{L}=3.2139 \times 10^{4} \Omega$. The value of $R / Q$ for the closed cavity is $(R / Q)_{c}=146.21$. The loaded $Q$ value can now be calculated from Eq. (21) to be $Q_{L}=219.8$. As noted earlier, for a high- $Q$ cavity the value of $Q_{L}$ calculated witlt the electromagnetic field approach is in good agreement with the equivalent circuit approach $\left(Q_{L}=210.0\right)$.

\section{Analysis of Low- $Q$ Cavities}

Much attention has been focused recently in the development of high-frequency, high-power cavities for applications such as future linear colliders and compact accelerators. It is therefore of particular interest to see the extent to which the methodologies discussed in the previous sections would be applicable to low- $Q$ cavities; nnd how these approaches could be used as computer aided tools in designing thrse cavitie's. We consider in the following two relativistic klystron ravities and present numerical calculations to compare with experimental measurements.

\section{Case 1. Relativistic hlystron Output Cavity with $Q_{L}=20$ (SL4)}

An output cavity designed by T. G. Lec for a relativistic klystron experiment* currently in progress at LLNL is shown in Fig. 7. The cavity coupled to a WR90 waveguide is designed to extract power at $11.4 \mathrm{GHz}$, and has been measured to have a loaded $Q$ value of 20 . For this low- $Q$ cavity the simple assumptions in the equjvalent circuit approach lead to rather larger discrepancy betwcen the calculated $Q_{L}$ and the measured value, since the consistency criterion which we have suggested before [Eq. (12)] for the equivalent circuit method is not met. We turn therefore to the electromagnetic field approach to calculate the loaded $Q$ for the output cavity.

a. For simplicity we first calculate the loaded $Q$ ignoring the small discontinuity in the WR90 wareguide. We have made two MAFIA runs using a relaiively coarse mesh for the cavity shown in Fig. 7 attached respectively to a 1.0-inch and a 0.8 -inch section of the WR90 waveguide. The results for the closed cavitywaveguide structures are shown in Table $I$, where $F$ is the normalized forward amplitude defined as:

$$
F=\left(\frac{1}{2}\right)\left(\frac{E_{\max }}{V}\right)=-16.343 \mathrm{~m}^{-1}
$$

where the normalization factor $V$ is given by Eq. (16).

* M. A. Allen el al., SLAC-PUB-4650, June 1988. 
Using the values in Table 1, we calculate the normalized forward intensity from Eq. (14) to be $|I|=30.370 \mathrm{~m}^{-1}$.

Table 1.

Parameters for SL4 Output Cavity $(\mathrm{Q}=20)$

with Coarse Mesh

\begin{tabular}{|l|c|c|}
\hline$f(\mathrm{GHz})$ & 11.1637 & 11.2991 \\
\hline$k\left(\mathrm{~cm}^{-1}\right)$ & 1.8916 & 1.9265 \\
\hline$\lambda(\mathrm{cm})$ & 3.3216 & 3.2614 \\
\hline$h(\mathrm{~cm})$ & 2.5400 & 2.0320 \\
\hline$\phi(\mathrm{cad})$ & 4.8047 & 3.9147 \\
\hline$E_{\text {max }}(V / \mathrm{m})$ & -0.1643 & -0.1857 \\
\hline$V(\mathrm{~V})$ & $5.027 \times 10^{-3}$ & $5.333 \times 10^{-3}$ \\
\hline$F\left(\mathrm{~m}^{-1}\right)$ & -16.343 & -17.411 \\
\hline
\end{tabular}

The shunt resistance $R$ related to the outgoing power flow, given by Eq. (19), is thus $4323 \Omega$.

The shunt resistance $R_{c}$ of the closed cavity is $5.45 \times 10^{5} \Omega$, and the loaded shunt resistance $R_{L}$, given by Eq. (20), is $4306 \Omega$.

Using the value of $(R / Q)_{c}=137.02 \Omega$, the loaded $Q_{L}$ is calculated to be $Q_{L}=$ $R_{L} /(R / Q)_{c}=31.4$.

b. The effect of the discontinuity in the waveguide (see Fig. 7) is estimated in Appendix A. Although each discontinuity introduced into the structure would cause an infinite number of reflections and hence contribute to the electromagnetic field at a given point, the net effect is to reduce the value of $Q_{L}$ only by about $2 \%$.

c. The accuracy of the above calculation can be improved by repeating the MAFIA runs with a finer mesh. The result for this set of runs are surnmarized in Table 2. A plot of the electric field in the closed cavity is shown in Fig. 8. The resulting value of $Q_{L}$ for the open cavity, considering only a single mode propagation, is 28.2, which compares favorably with the measured value. 
Table 2.

Parameters for SL4 Output Cavity $(Q=20)$ with Fine Mesh

\begin{tabular}{|l|c|c|}
\hline$f(\mathrm{GHz})$ & 11.2113 & 11.3760 \\
\hline$k\left(\mathrm{~cm}^{-1}\right)$ & 1.9039 & 1.9463 \\
\hline$\lambda(\mathrm{cm})$ & 3.3001 & 3.2283 \\
\hline$h(\mathrm{~cm})$ & 2.5400 & 2.0320 \\
\hline$\phi(\mathrm{rad})$ & 4.8359 & 3.9549 \\
\hline$E_{\max }(\mathrm{V} / \mathrm{m})$ & -0.2108 & -0.2043 \\
\hline$V(\mathrm{~V})$ & $5.140 \times 10^{-3}$ & $5.538 \times 10^{-3}$ \\
\hline$F\left(\mathrm{~m}^{-1}\right)$ & -20.516 & -18.446 \\
\hline
\end{tabular}

d. In principle, all modes capable of propagating in the waveguide contribute to the power loss. The effect of modes other than the TE mode which we have calculated above can be estimated by examining the relative field amplitudes to $E_{2}$. For the relativistic klystron output cavity, other field components are small. Thus the single mode dominance in the power extraction is a reasonable assumption. Althougl our methodology can be applied in a straightforward way to other modes, the computations could be tedious if many modes contribute to the value of $Q_{L}$.

Case 2. Relativistic Klystron Output Cavity with $Q=40$

Next we consider another low- $Q$ output cavity tested in the relativistic klystron experiment with a subharmonic drive $(5.7 \mathrm{GHz})$ at LLNL. This cavity, designed to extract lower power at $11.4 \mathrm{Gllz}$, has a measured value of loaded $Q$ of 40 . The dimensions of the cavity are shown in Fig. 9. Again, with two MAFIA rums at waveguide lengths of 1.0 -inch and 0.8 -inch, we calculated a loaded $Q_{L}$, value of 43.7 .

\section{APPENDIX A: EFFECT OF WAVEGUIDE DISCONTINUITY ON $Q_{L}$}

To estimate the effect of the discontinuity in the waveguide for the $Q=20$ relativistic klystron output cavity, we assume that the transmission coefficients from regions 1 to $2, t_{1}$, and from 2 to $1, t_{2}$, are of the fundamental mode. We further assume that the transmission coefficients are larger than the reflection coefficients $R_{1}$ and $B_{2}$. Therefore, rays transmitted through both regions will be dominant. The first order correction to these diagrams caused by reflection at the discontinuity is given by 


$$
\exp (i k y) F=\frac{I}{1+\rho_{1} \cdot t_{1} t_{2} \exp \left(-2 i \phi_{1}\right)}\left\{1+\frac{\exp \left[-2 i\left(\phi_{1}+\phi_{2}\right)\right] R_{2} \cdot t_{1} t_{2} \rho_{1}}{1+R_{2} \cdot \exp \left(-2 i \phi_{2}\right)}\right\}
$$

where we use the notation in Eq. (14) and the phase angles are given by:

$$
\begin{aligned}
& \phi_{1}=k h \\
& \phi_{2}=k(h-d) .
\end{aligned}
$$

where $d$ is the distance to discontinuity and $h$ is the distance to termination of the waveguide.

Denoting by

$$
\hat{\rho}_{1}=\rho_{1} \cdot t_{1} \cdot t_{2}
$$

we can write Eq. (A.I):

$$
\exp (i k y) \cdot F=\frac{I}{1+\hat{\rho}_{1} \exp \left(-2 i \phi_{j}\right)}\left\{1+\frac{\exp \left[-2 i\left(\phi_{1}+\phi_{2}\right)\right] \hat{\rho}_{1} R_{2}}{1+R_{2} \cdot \exp \left(-2 i \phi_{2}\right)}\right\} \quad
$$

Equation (A.4) has three unknowns, $I, \hat{p}_{1}$ and $R_{2}$. To solve for these parameters, we have to sun the MAFIA code to simulate the cavily with three different waveguide sections. We finally end up with three complex algebraic er, tations:

$$
-\frac{i}{A_{j}} F_{j}=\frac{I}{1+\hat{\rho}_{1} A_{j}^{2}}\left(1+\frac{A_{j}^{2} B_{j}^{2} \widehat{p}_{1} R_{2}}{1+\frac{B_{j}^{2} R_{2}}{R^{2}}}\right)
$$

where

$$
\begin{aligned}
A_{j} & =\exp \left[-i \phi_{j}^{(j)}\right], \\
B_{j} & =\exp \left[-i \phi_{2}^{(j)}\right], \\
j & =1,2,3
\end{aligned},
$$

and $F_{j}$ relates, respeclively, to the three runs with different waveguide sections. In Eq. (A.5) we make use of the relation:

$$
y=h-\frac{\lambda}{4}
$$

The first order correction of the discontinuity to the output radiation astuplitude is given by:

D. Yu/January 1989 


$$
I=-i \frac{F_{3}}{A_{3}} \frac{1-\left(A_{3}^{2} / A_{1}^{2}\right)}{1-\left(F_{3} A_{3} / F_{1} A_{1}\right)}\left[1+\frac{\epsilon}{1-\left(F_{3} A_{3} / F_{1}\right.} \frac{}{\left.A_{1}\right)}\right]
$$

where

$$
\begin{gathered}
c=\frac{A_{3}^{2}}{A_{1}^{2}}\left(i \frac{I_{0} A_{1}}{F_{1}}-1\right) \frac{X-1}{\left(A_{1} / F_{1}\right) B_{1}^{d}-\left(A_{2} / F_{2}\right) B_{2}^{2}} \\
\cdot\left\{i \frac{B_{3}^{2}}{I_{0}}+\frac{\left(A_{1} / F_{1}\right) B_{1}^{2}}{1+\left(A_{3}^{2}\right.} \frac{\left.\sqrt{\left.A_{1}^{2}\right)\left[i\left(I A_{1} / F_{1}\right)-1\right]}\right\}}{I_{0}=-i \frac{F_{3}}{A_{3}} \frac{1-\left(A_{3}^{2} / A_{1}^{2}\right)}{1-\left(F_{3} A_{3} / F_{1} A_{1}\right)}}\right. \\
X=\frac{A_{2}^{2}}{A_{1}^{2}} \cdot \frac{i\left(I_{0} A_{1} / F_{1}\right)-1}{i\left(I_{0} A_{2} / F_{2}\right)-1} \quad \text { for }|X-1|<1
\end{gathered}
$$

Substituting the parameters for waveguide sections of 1.0 -inch, 0.9 -inch and 0.8 -inch, we end with loaded $Q_{L}$ for a coarse mesh:

$$
Q_{L}=30 ., 0
$$

which is only slightly different from the value without the discontinuity:

$$
Q_{L}=31.43
$$

Running the same problem with a finer mesh further reduced the $Q_{L}$ value. With this mesh we obtain:

$$
Q_{L}=28.21
$$

\section{DISCLAIMER}

This report was prepared as an eccownt of wotk sponsored by an agency of the Unitod Stales Government. Neither the United States Government nor uny wency thereor, nor any of their employees, makes any warrenty, express or implied, or assumes any legal liability or responsibility for the excuracy, compleleneas, ar usefuliness of any information, apparatus, product, or process disclosed, or represents that its use would not infringe privately owned rights. Reference acrein to any specific cothmercial product, process, of service by trade name, traderuart. manufacturer, of otherwice dos not aecessarily constitute or imply its endorsement, recommendation, or favoring by the Unjed Siates Govemment of any agency thereof. The views and opinions of authors expresed herein do not necessarily state or reflect these of the United Sintes Government or any agency thereof. 


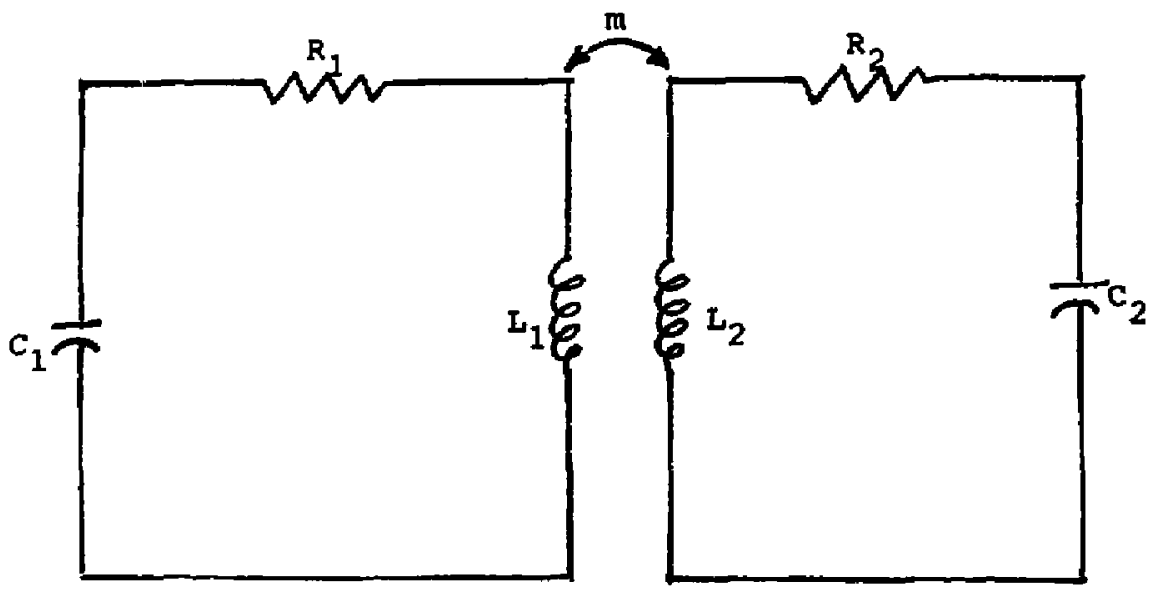

Fig. 1. Equivalent circuit for closed waveguide-cavity. 


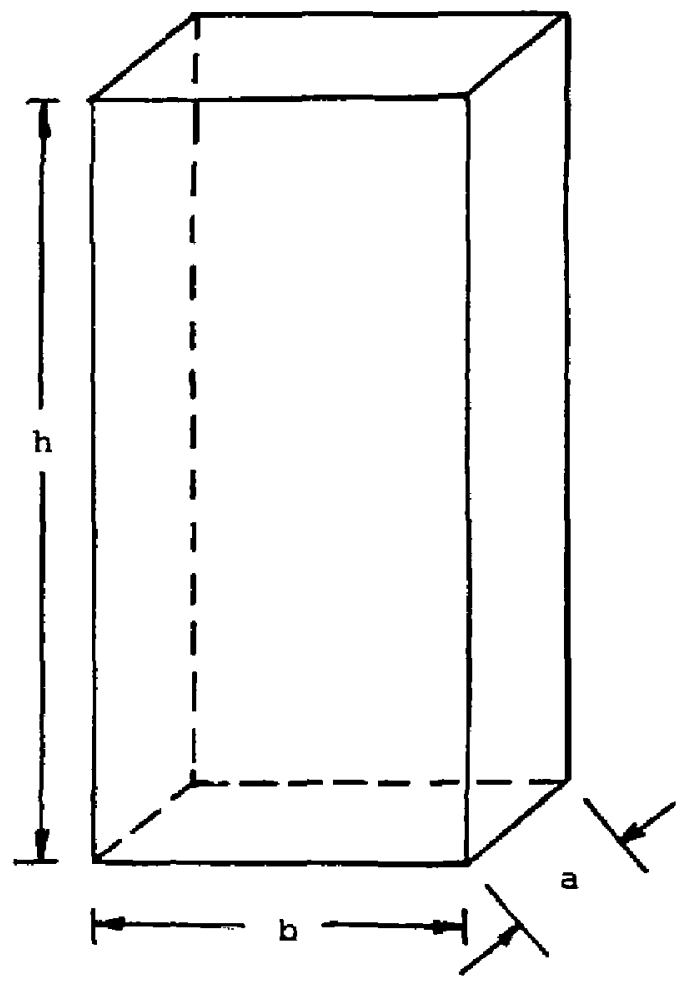

Fig. 2. Rectangular waveguide dimensions. 


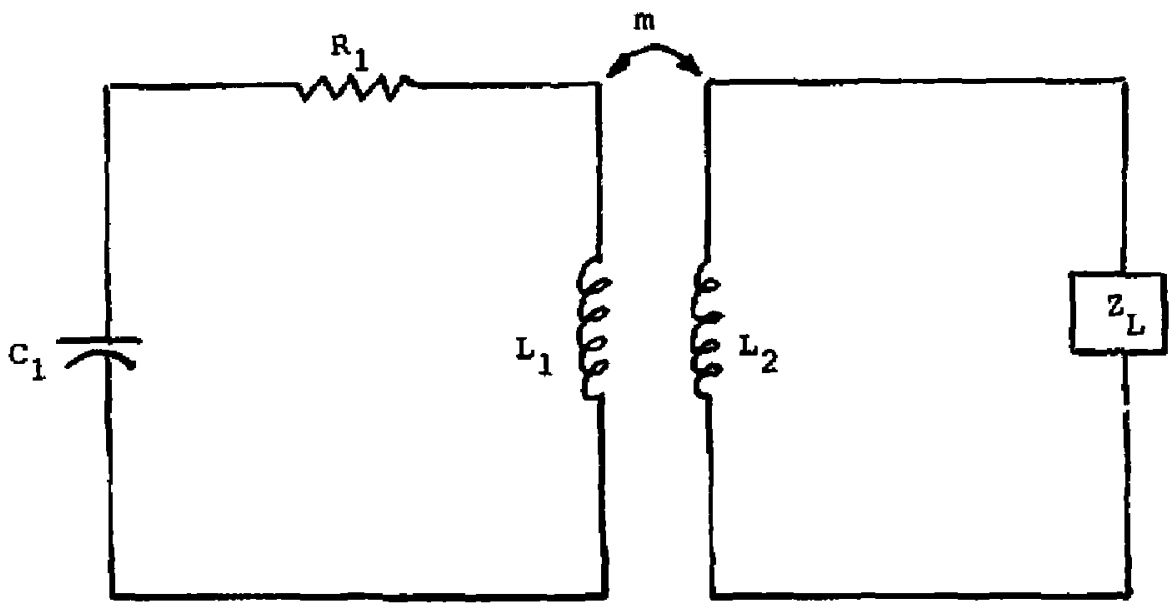

Fig. 3. Equivalent circuit for opened uraveguide-cavity: 


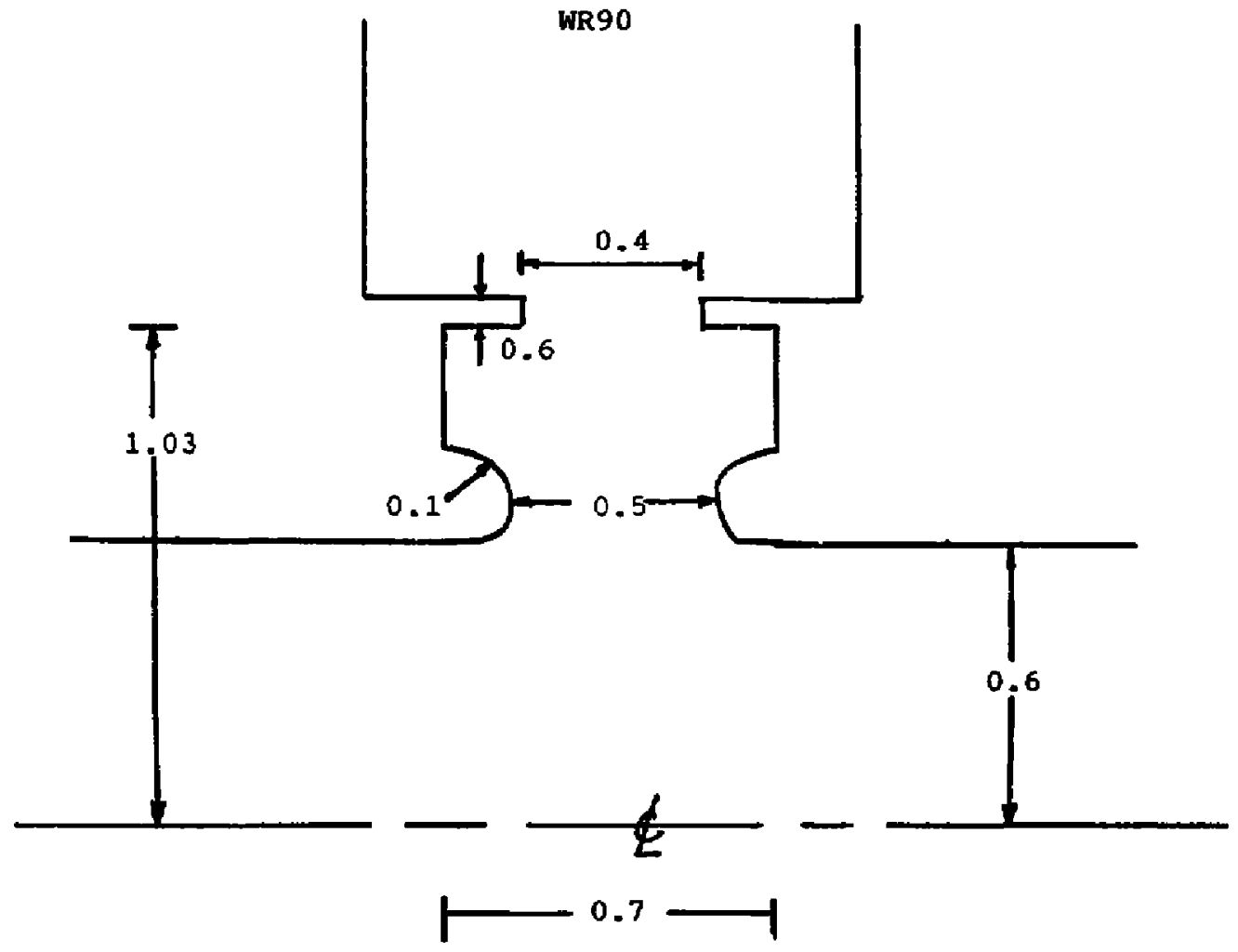

Fig. 4. Dimensions (in $\mathrm{cm}$ ) of a high- $Q_{L}$ cavity-waveguide coupler. 


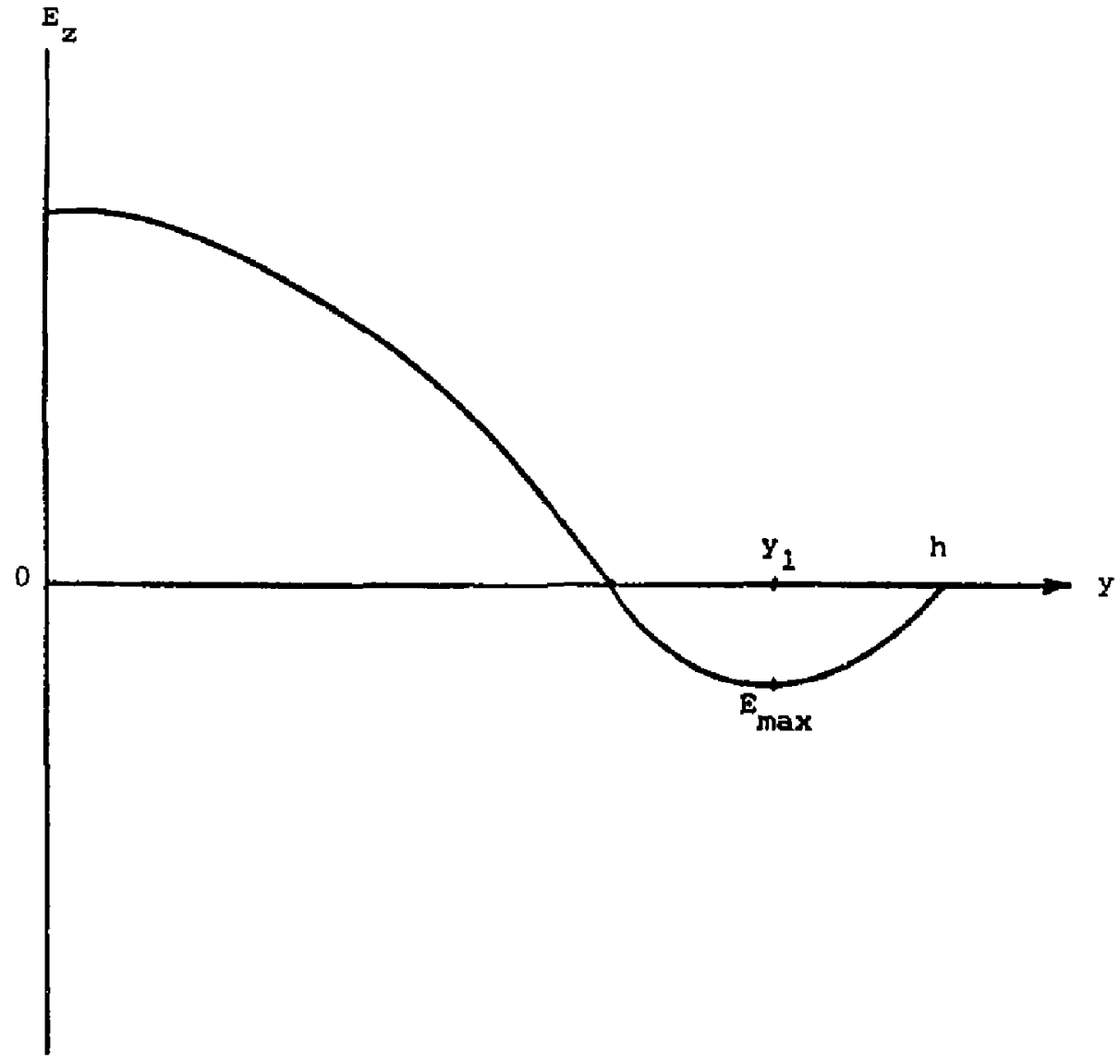

Fig. 5. Standing-wave pattern in wat guide. 


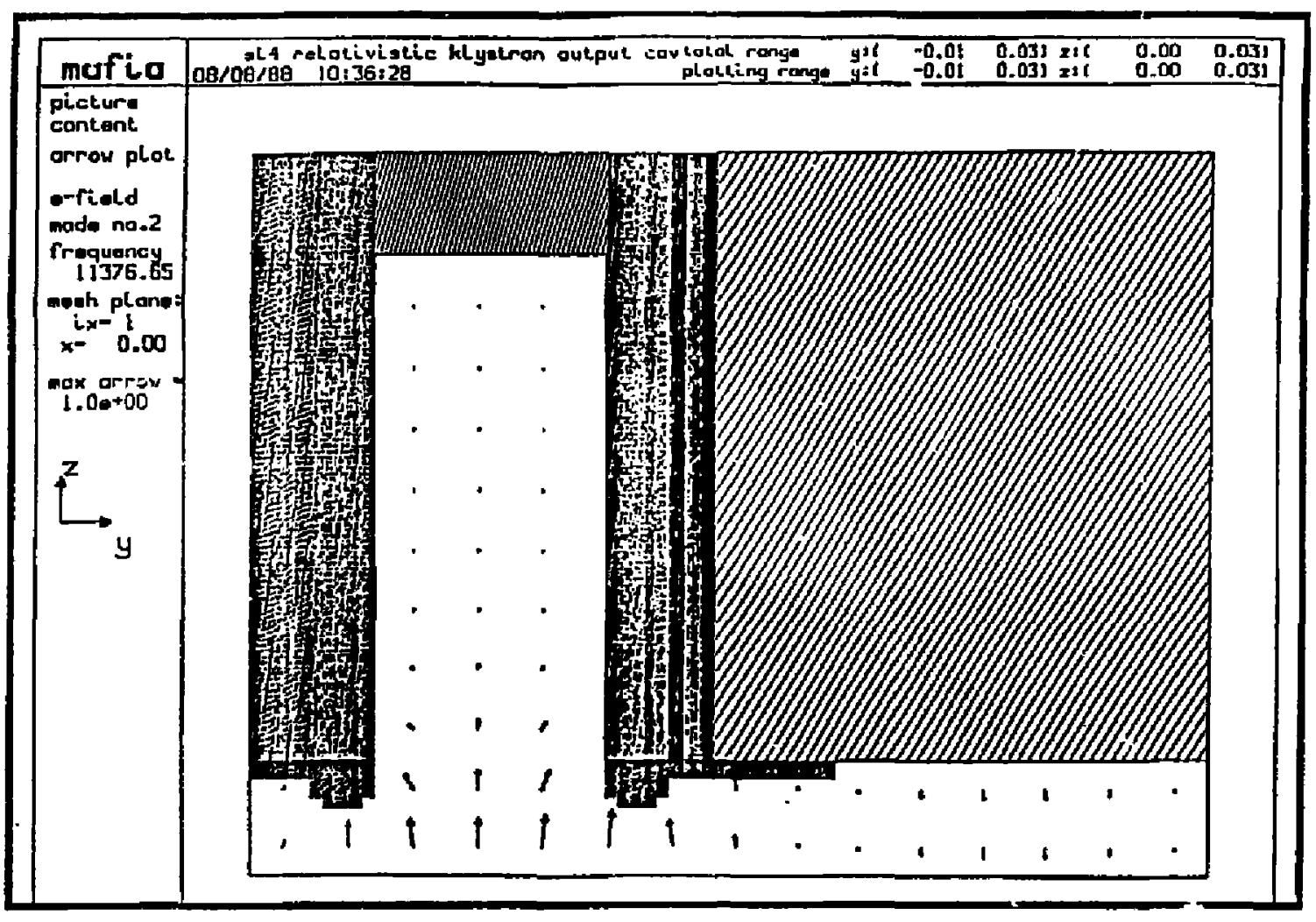

Fig. 6. MAFIA electric field lines. 


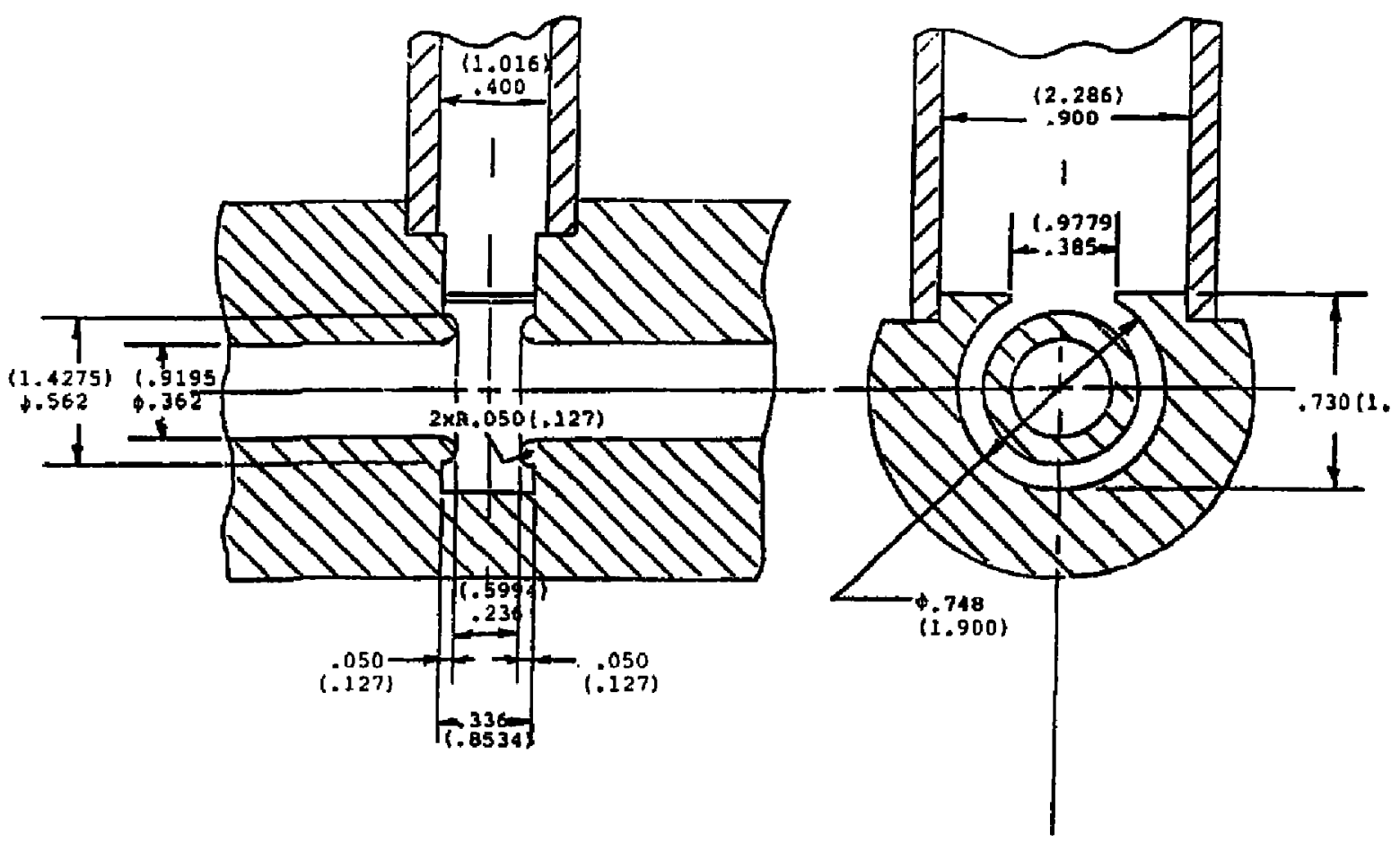

Fig. 7. Relativistic klystron SL4 output cavity with $Q_{L}=20$ (dimensions in inches $(\mathrm{cm})$. 


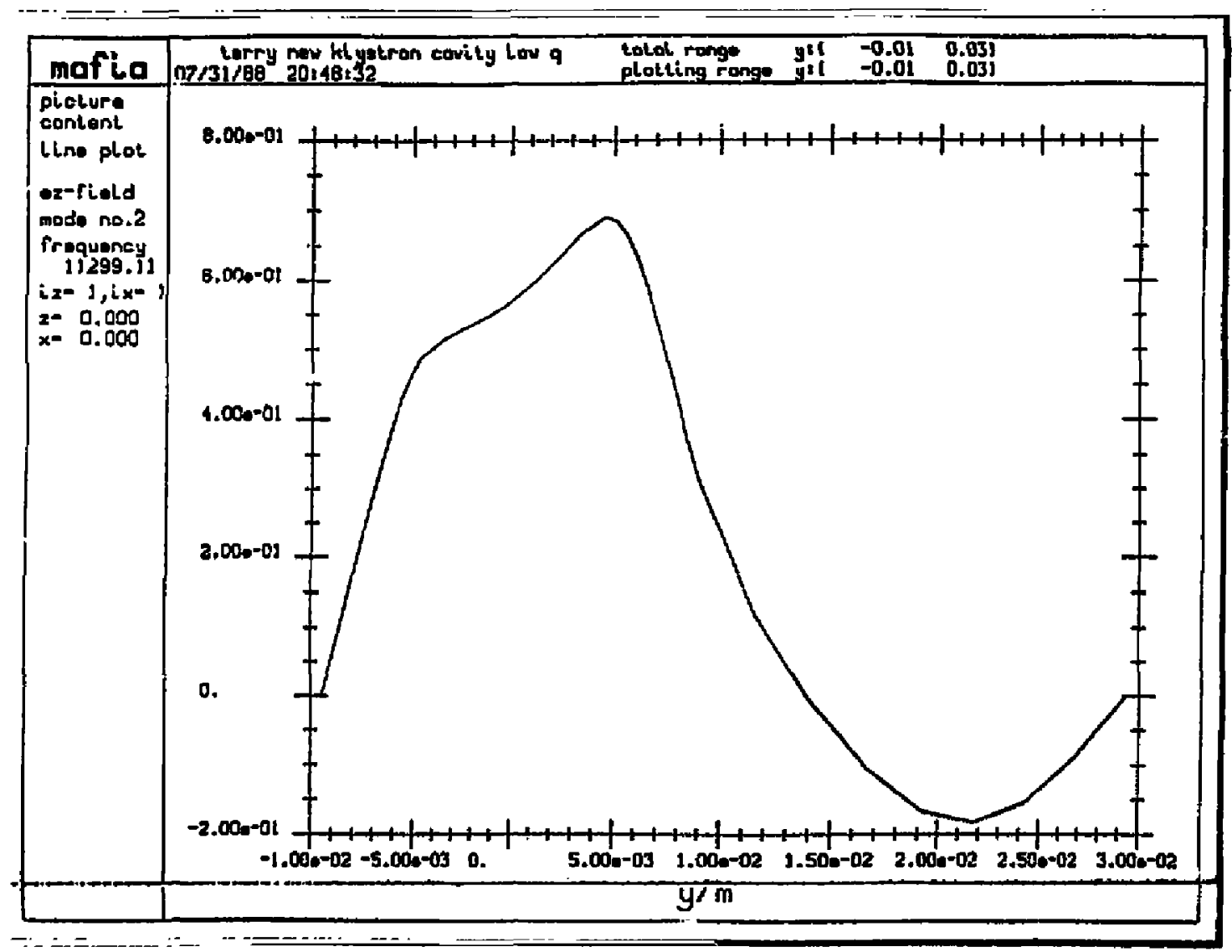

Fig. 8. $E_{z}$ versus $y$ plot for $Q_{L}=90$ cavity. 


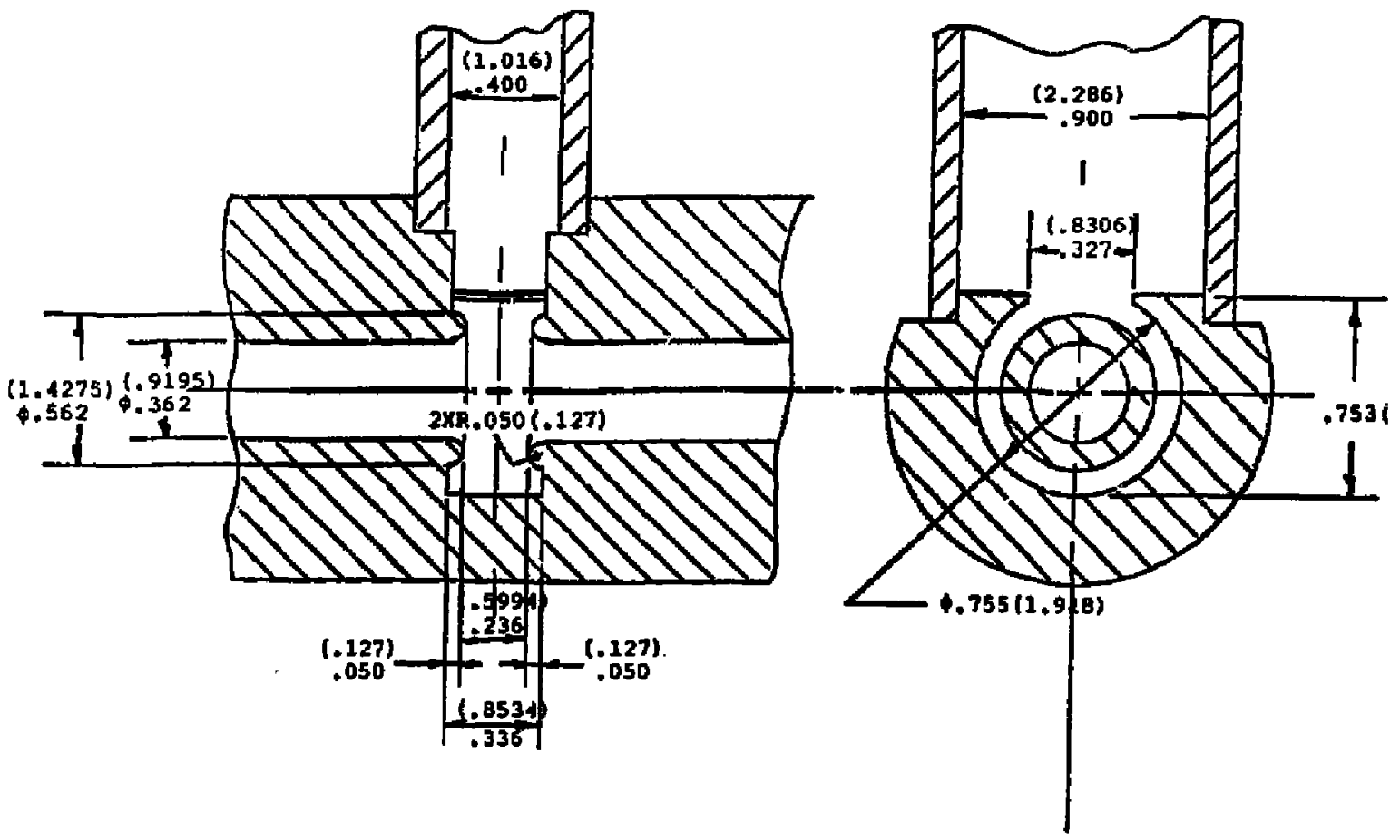

Fig 9. Relativistic klystron SHARK output cavity with $Q_{L}=40$. 